PROCEEDINGS OF THE

AMERICAN MATHEMATICAL SOCIETY

Volume 139, Number 4, April 2011, Pages 1141-1154

S 0002-9939(2010)10776-1

Article electronically published on November 17, 2010

\title{
EMBEDDING PROBLEMS AND OPEN SUBGROUPS
}

\author{
DAVID HARBATER AND KATHERINE STEVENSON
}

(Communicated by Ted Chinburg)

\begin{abstract}
We study the properties of the fundamental group of an affine curve over an algebraically closed field of characteristic $p$ from the point of view of embedding problems. In characteristic zero the fundamental group is free, but in characteristic $p$ it is not even $\omega$-free. In this paper we show that it is "almost $\omega$-free" in the sense that each finite embedding problem has a proper solution when restricted to some open subgroup. We also prove that embedding problems can always be properly solved over the given curve if suitably many additional branch points are allowed in locations that can be specified arbitrarily; this strengthens a result of the first author.
\end{abstract}

\section{INTRODUCTION}

This paper studies the structure of the étale fundamental group of an affine curve over an algebraically closed field of characteristic $p$ and shows that in a certain sense it is "not too far from being free."

Consider an affine curve $C$ over an algebraically closed field $k$. Thus $C=X-\Sigma$, where $X$ is a smooth projective curve of genus $g \geq 0$ and $|\Sigma|=r>0$. If $k$ has characteristic zero, then $\pi_{1}(C)$ is a free profinite group on $2 g+r-1$ generators SGA1, XIII, Cor. 2.12]. In contrast, if $k$ has characteristic $p>0$, then $\pi_{1}(C)$ is infinitely generated as a profinite group, since by Artin-Schreier theory there are infinitely many linearly disjoint $\mathbb{Z} / p \mathbb{Z}$-Galois étale covers of $C$. Moreover it cannot be free profinite (of infinite rank), since a prime-to- $p$ group is a quotient of $\pi_{1}(C)$ if and only if it has at most $2 g+r-1$ generators [SGA1, XIII, Cor. 2.12]). For this same reason, $\pi_{1}(C)$ is not even $\omega$-free; i.e. it is not the case that every finite embedding problem for $\pi_{1}(C)$ has a proper solution [FJ, p. 652]. (Infinitely generated free profinite groups are $\omega$-free [FJ, Lemma 25.1.2]; the converse holds for countably generated groups [Iw, p. 167], but not in general [Ja, Example 3.1].)

The main result of this paper (Theorem 6) is to show that $\Pi=\pi_{1}(C)$ has the weaker property of being almost $\omega$-free: for every finite embedding problem $\mathcal{E}=(\alpha: \Pi \rightarrow G, f: \Gamma \rightarrow G)$ there is an open subgroup $H \subset \Pi$ such that

Received by the editors December 6, 2009.

2010 Mathematics Subject Classification. Primary 14G17, 14H30, 20E18; Secondary 12E30, 14G32, 20F34.

Key words and phrases. Fundamental group, affine curve, characteristic $p$, embedding problem, omega-free.

The first author was supported in part by NSF grant DMS-0901164.

The second author was supported in part by NSF grant IIS-0534984.

(C)2010 American Mathematical Society 
$\left.\alpha\right|_{H}: H \rightarrow G$ is surjective and the induced embedding problem $\mathcal{E}_{H}=\left(\left.\alpha\right|_{H}, f\right)$ has a proper solution.

This result can be interpreted geometrically as follows. We are given a $G$-Galois cover $\phi: Y \rightarrow X$ ramified at most over $\Sigma$. A proper solution to the original embedding problem is a $\Gamma$-Galois cover $Z \rightarrow X$ that dominates $\phi: Y \rightarrow X$ and is unramified away from $\Sigma$. The almost $\omega$-free property asserts that there exists a cover $\psi: X^{\prime} \rightarrow X$ ramified at most over $\Sigma$ such that the normalized pullback $\phi^{\prime}: Y^{\prime} \rightarrow X^{\prime}$ of $\phi$ via $\psi$ is a $G$-Galois cover and moreover there exists a $\Gamma$-Galois cover $Z^{\prime} \rightarrow X^{\prime}$ that dominates $Y^{\prime} \rightarrow X^{\prime}$ and is branched only at points of $X^{\prime}$ lying over $\Sigma \subset X$. Here the cover $\psi$ corresponds to the restriction $\left.\alpha\right|_{H}$, where $H \subset \pi_{1}(X-\Sigma)$ is the open subgroup $\pi_{1}\left(X^{\prime}-\psi^{-1}(\Sigma)\right)$.

This paper is motivated by the fact that the absolute Galois group $G_{k(X)}$ of the function field of a smooth projective $k$-curve $X$ is free, for $k$ an algebraically closed field of arbitrary characteristic ( $\left.\mathrm{Ha}_{95}, \mathrm{PO}\right)$. In characteristic zero this is natural since each $\pi_{1}(C)$ is free, and in that case the freeness of $G_{k(X)}$ was proven earlier by viewing this group as the inverse limit of the free groups $\pi_{1}(C)$ for dense affine open subsets $C \subset X\left[\mathrm{Do}\right.$. But in non-zero characteristic, the freeness of $G_{k(X)}$ is a bit mysterious, since the groups $\pi_{1}(C)$ are not free. Still, this suggests that the groups $\pi_{1}(C)$ should satisfy some kind of freeness property, and this role is played here by the property of being almost $\omega$-free. (The word almost is being used in the sense of "up to a finite discrepancy"; here the open subgroup $H$ is necessarily of finite index.)

In the process of proving our main theorem, we also obtain a result (Theorem 5) that strengthens the main theorem of [Ha99. That theorem, which provided a more precise form of a key result in [Ha95] and $\mathrm{Po}$, had asserted that an embedding problem for an affine curve over an algebraically closed field can be solved if a specific number of additional points are permitted. Our result here shows that the positions of the additional points can be chosen arbitrarily.

We also note the papers $\mathrm{Ku} 08$ and $\mathrm{Ku} 09$, which discuss another way in which $\pi_{1}(C)$ is "close to being free". Namely, those papers show that if $k$ is an algebraically closed field of characteristic $p$, then the commutator subgroup of $\pi_{1}(C)$ is free. For other papers on related themes, see also $\mathrm{Bo}, \mathrm{GS}, \mathrm{Os}, \mathrm{Pr}, \mathrm{Ta}$.

The structure of the current manuscript is as follows: In Section 2 we prove Proposition 2, which asserts that $G$-Galois branched covers have pullbacks that remain $G$-Galois but become unramified at sufficiently many points lying over the original branch locus. Section 3 proves results leading up to Theorem 5 on the solution to embedding problems with prescribed additional branch points. These are combined in Section 4 to obtain our main result, Theorem 6, that the fundamental group is almost $\omega$-free. That section also discusses almost $\omega$-freeness in the characteristic zero case, where the fundamental group is finitely generated.

Terminology and Notation: If $G$ is a finite group and $p$ is a prime number, let $p(G)$ denote the subgroup of $G$ generated by its $p$-subgroups. This is a characteristic subgroup of $G$, and $G / p(G)$ is the maximal prime-to-p quotient of $G$. A finite group $G$ is called quasi-p if $G=p(G)$. A finite embedding problem $\mathcal{E}$ for a group $\Pi$ is a pair of surjections $(\alpha: \Pi \rightarrow G, f: \Gamma \rightarrow G)$, where $\Gamma$ and $G$ are finite groups. A weak solution to $\mathcal{E}$ is a homomorphism $\gamma: \Pi \rightarrow \Gamma$ such that $f \circ \gamma=\alpha$. We call $\gamma$ a proper solution to $\mathcal{E}$ if in addition it is surjective. If $H$ is a subgroup of $\Pi$ and 
$\left.\alpha\right|_{H}$ is a surjection onto $G$, then $\mathcal{E}^{\prime}=\left(\left.\alpha\right|_{H}: H \rightarrow G, f: \Gamma \rightarrow G\right)$ is the induced embedding problem for $H$.

In this paper we consider curves over an algebraically closed field $k$. A cover of $k$-curves is a morphism $\phi: V \rightarrow U$ of smooth connected $k$-curves that is finite and generically separable. If $\phi: V \rightarrow U$ is a cover, its Galois group $\operatorname{Gal}(V / U)$ is the group of $k$-automorphisms $\sigma$ of $V$ satisfying $\phi \circ \sigma=\phi$. If $G$ is a finite group, then a G-Galois cover is a cover $\phi: V \rightarrow U$ together with an inclusion $\rho: G \hookrightarrow \operatorname{Gal}(V / U)$ such that $G$ acts simply transitively on a generic geometric fibre of $\phi: V \rightarrow U$. If we fix a base point of $U$, then the pointed $G$-Galois étale covers of $U$ correspond bijectively to the surjections $\alpha: \pi_{1}(U) \rightarrow G$, where $\pi_{1}(U)$ is the algebraic fundamental group of $U$. The proper solutions to an embedding problem $\mathcal{E}=\left(\alpha: \pi_{1}(U) \rightarrow G, f: \Gamma \rightarrow G\right)$ for $\pi_{1}(U)$ then are in bijection to the pointed $\Gamma$-Galois covers $W \rightarrow U$ that dominate the pointed $G$-Galois cover $\phi: V \rightarrow U$ corresponding to $\alpha$.

\section{REMOVING BRANCH POINTS BY PULLING BACK}

Fix an algebraically closed field $k$ of characteristic $p>0$. The goal of this section is to prove Proposition 2 below, which says that for any branched cover of $k$-curves, there is a finite morphism to the base such that the inverse image of the original branch locus contains (many) points that are unramified in the normalized pullback of the given cover. This will be used in conjunction with the key result of the next section in order to prove our main theorem. The point is that since affine curves are not $\omega$-free, not all embedding problems can be properly solved; but by proceeding to a pullback via Proposition 2 we may enlarge the branch locus and thereby obtain a solution to the induced embedding problem.

Lemma 1. Let $X$ be a smooth connected projective $k$-curve and let $\Delta \subset X$ be a non-empty subset of $X$. Given an integer $t>1$ and a G-Galois cover $\phi: Y \rightarrow X$ étale away from $\Delta$, there is a Galois cover $\pi: X_{0} \rightarrow X$ of degree at least $t$ that is linearly disjoint from $Y \rightarrow X$, is branched only over $\Delta$, and such that $\left|\pi^{-1}(\Delta)\right| \geq t$.

Proof. Fix a point $\sigma \in \Delta$, and choose an integer $n \geq \max (5, p)$ such that $A_{n}$ is not a quotient of $G$ and the largest prime-to- $p$ factor $m$ of $\left|A_{n}\right|$ is at least $t$. The group $A_{n}$ is simple, so every $A_{n}$-Galois cover of $X$ is linearly disjoint from $Y$ over $X$ having no common subcovers.

Since $A_{n}$ is simple of order divisible by $p$, it is a quasi- $p$-group. So by Abhyankar's Conjecture [Ha94, Theorem 6.2] (or by [Ra, Corollaire 2.2.2], in the case of the affine line), there is an $A_{n}$-Galois cover $\pi: X_{0} \rightarrow X$ that is branched only at $\sigma \in X$. Moreover, this cover may be chosen so that the inertia groups over $\sigma$ are the Sylow $p$-subgroups of $A_{n}$ by [Po, Theorem B] (see also [Ha03a, Corollary 4.2]). The number of ramification points over $\sigma$ is the index of such a Sylow $p$-subgroup $Q$. Since the index $m$ of $Q$ in $A_{n}$ is greater than or equal to $t$, the set $\pi^{-1}(\sigma)$ contains at least $t$ points, and hence so does $\pi^{-1}(\Delta)$, which contains it.

Proposition 2. Let $X$ be a smooth connected projective $k$-curve, let $\Sigma \subset X$ be a non-empty subset of $X$, and fix a point $\tau$ in $\Sigma$. Let $G$ be a finite group and let $\phi: Y \rightarrow X$ be a $G$-Galois cover that is étale away from $\Sigma$. Then for every positive integer $s$ there is a cover $\psi_{s}: X_{s} \rightarrow X$ that is étale away from $\Sigma$ and is linearly disjoint from $\phi: Y \rightarrow X$ such that the normalized pullback $\phi_{s}: Y_{s} \rightarrow X_{s}$ of $\phi$ via $\psi_{s}$ is a $G$-Galois cover with at least s unbranched points in $\psi_{s}^{-1}(\tau) \subset X_{s}$. 
Proof. Applying Lemma 1 to the cover $\phi: Y \rightarrow X$ with $t=2$ and $\Delta=\Sigma$, there is a Galois cover $\pi: X_{0} \rightarrow X$ of degree $d \geq 2$ that is linearly disjoint from $\phi: Y \rightarrow X$, is étale away from $\Sigma$ and such that $\left|\pi^{-1}(\Sigma)\right| \geq 2$. By linear disjointness, the normalized pullback $Y_{0} \rightarrow X_{0}$ of $Y \rightarrow X$ is connected, and so it is a $G$-Galois cover. Replacing $Y \rightarrow X$ by $Y_{0} \rightarrow X_{0}$ and $\Sigma$ by $\pi^{-1}(\Sigma)$, we may assume that $|\Sigma| \geq 2$.

Next we reduce to the case that $s=1$. Suppose that there exists a cover $\psi_{1}: X_{1} \rightarrow X$ étale away from $\Sigma$ and linearly disjoint from $\phi: Y \rightarrow X$ such that the normalized pullback $\phi_{1}: Y_{1} \rightarrow X_{1}$ of $\phi$ via $\psi_{1}$ contains at least one point $\tau_{1}$ in $\psi_{1}^{-1}(\tau)$ that is unbranched in $\phi_{1}$. Necessarily $Y_{1}$ is connected by linear disjointness. Let $\Delta=\psi_{1}^{-1}(\Sigma)-\left\{\tau_{1}\right\}$. This is non-empty by the assumption that $|\Sigma| \geq 2$. Applying Lemma 1 again, but this time to the cover $\phi_{1}$ with $t=s$, there is a cover $\pi_{s}: X_{s} \rightarrow X_{1}$ such that its degree is at least $s$; it is linearly disjoint from $\phi_{1}: Y_{1} \rightarrow X_{1}$, it is étale away from $\Delta$, and $\left|\pi_{s}^{-1}(\Delta)\right| \geq s$. Consider the normalized pullback $\phi_{s}: Y_{s} \rightarrow X_{s}$ of $\phi_{1}$ via $\pi_{s}$; this is a connected $G$-Galois cover because $X_{s}$ and $Y_{1}$ are linearly disjoint over $X_{1}$. Moreover, as $\tau_{1} \notin \Delta$, the morphism $\pi_{s}$ is unramified over $\tau_{1}$. But $\pi_{s}$ has degree at least $s$, and so $\pi_{s}^{-1}\left(\tau_{1}\right)$ contains at least $s$ points. Each point in $X_{s}$ lying over $\tau_{1}$ is unbranched in $\phi_{s}$, since $\tau_{1}$ is unbranched in $\phi_{1}$. Let $\psi_{s}:=\psi_{1} \circ \pi_{s}: X_{s} \rightarrow X$. Thus $\phi_{s}: Y_{s} \rightarrow X_{s}$ is the normalized pullback of $\phi: Y \rightarrow X$ via $\psi_{s}$, and $X_{s}$ is linearly independent of $Y$ over $X$ by connectivity of $Y_{s}$. Finally, since $\tau_{1}$ lies over $\tau$ in $X_{1}$, we have that $X_{s}$ contains at least $s$ points in $\psi_{s}^{-1}(\tau) \supset \pi_{s}^{-1}\left(\tau_{1}\right)$ that are unbranched in $\phi_{s}$. Thus the cover $\psi_{s}: X_{s} \rightarrow X$ has the desired properties.

It now remains to prove the result for $s=1$. We consider three cases.

Case 1. The inertia groups of $Y \rightarrow X$ over $\tau$ are non-trivial $p$-groups, and there is a point $\sigma \in \Sigma-\{\tau\}$ such that the inertia groups of $\phi: Y \rightarrow X$ over $\sigma$ are the same as those over $\tau$.

Pick a point $\eta \in Y$ over $\tau$ and let $P \subset G$ be its inertia group. According to Proposition 2.7 of [Ha80, there is a $P$-Galois cover $W \rightarrow X$ that is unramified away from $\tau$, totally ramified at $\tau$, and such that the $P$-Galois extensions $\hat{\mathcal{O}}_{W, \omega}$ and $\hat{\mathcal{O}}_{Y, \eta}$ of $\hat{\mathcal{O}}_{X, \tau}$ are isomorphic, where $\omega$ is the unique point of $W$ over $\tau$. (In fact, according to the result cited, the number of such covers $W \rightarrow X$ is equal to the order of $H^{1}(X, P)$, though we do not need this here.)

Since $Y \rightarrow X$ has the same sets of inertia groups over $\tau$ and $\sigma$, whereas $W \rightarrow X$ is totally ramified over $\tau$ and unramified over $\sigma$, it follows that these two Galois covers are linearly disjoint (having no common subcovers). So the normalized pullback $\phi_{W}: Y_{W} \rightarrow W$ of $Y \rightarrow X$ via $W \rightarrow X$ is an irreducible $G$-Galois cover. This cover is unramified at $\omega$, since it is trivial over the fraction field of $\hat{\mathcal{O}}_{W, \omega}$ (by the above isomorphism). Let $X_{1}=W, Y_{1}=Y_{W}$, and $\phi_{1}$ be the cover $\phi_{W}$. Now, $\omega \in W=X_{1}$ lies over $\tau \in X$. Thus $\psi_{1}: X_{1} \rightarrow X$ is étale away from $\Sigma$ and is linearly disjoint from $\phi: Y \rightarrow X$, and the normalized pullback $\phi_{1}: Y_{1} \rightarrow X_{1}$ contains at least one point in $\psi_{1}^{-1}(\tau)$ that is unbranched in $\phi_{1}$.

Case 2. There are distinct points $\sigma_{1}, \sigma_{2} \in \Sigma-\{\tau\}$ such that the inertia groups of $\phi: Y \rightarrow X$ over each $\sigma_{i}$ are the same as those over $\tau$.

Pick a point over $\tau$ and let $I$ be its inertia group. Then $I$ is a semi-direct product of a $p$-group $P$ and a cyclic prime-to- $p$ group $C=\langle\gamma\rangle$. The other inertia groups over $\tau$ (and hence over $\sigma_{1}$ and $\sigma_{2}$ ) are conjugate to $I$. Let $g$ be the genus of $X$. 
By [SGA1, XIII, Cor. 2.12], the prime-to- $p$ fundamental group $\pi_{1}^{\prime}$ of $X-\left\{\tau, \sigma_{1}\right\}$ has generators $a_{i}, b_{i}, c_{j}$ with $1 \leq i \leq g$ and $j=0,1$, subject to the single relation $\left(\prod_{i}\left[a_{i}, b_{i}\right]\right) c_{0} c_{1}=1$.

Take the surjection $\pi_{1}^{\prime} \rightarrow C$ given by sending each $a_{i}$ and $b_{i}$ to 1 , sending $c_{0}$ to $\gamma$, and sending $c_{1}$ to $\gamma^{-1}$. The corresponding $C$-Galois cover $\psi_{T}: T \rightarrow X$ is branched just at $\tau$ and $\sigma_{1}$, where it is totally ramified. Each non-trivial subcover $T^{\prime} \rightarrow X$ of $T \rightarrow X$ will also be totally ramified at $\tau$ and $\sigma_{1}$ and unramified at $\sigma_{2}$. On the other hand, each non-trivial subcover of $Y \rightarrow X$ will be branched at $\sigma_{2}$ if it is branched at $\tau$ and $\sigma_{1}$, because those three points have the same sets of inertia groups. So $T$ is linearly disjoint from $Y$ over $X$. Hence the normalized pullback $\phi_{T}: Y_{T} \rightarrow T$ of $Y \rightarrow X$ via $T \rightarrow X$ is an irreducible $G$-Galois cover. Moreover at the unique points $\tau^{\prime}, \sigma_{1}^{\prime} \in T$ over $\tau, \sigma_{1} \in X$, the inertia groups of $Y_{T} \rightarrow T$ have no tame part, by Abhyankar's Lemma. Also, the Galois group $C$ of $T \rightarrow X$ has order prime to $p$, whereas $P$ is a $p$-group. Hence the inertia groups of $Y_{T} \rightarrow T$ over $\tau^{\prime}, \sigma_{1}^{\prime}$ are isomorphic to $P$, and this cover is totally ramified over those two points.

If $P$ is trivial, then the cover $\phi_{T}: Y_{T} \rightarrow T$ is unbranched over the unique point $\tau^{\prime} \in T$ lying over $\tau \in X$. Taking $\psi_{s}: X_{s} \rightarrow X$ to be $\psi_{T}: T \rightarrow X$, the normalized pullback $\phi_{T}: Y_{T} \rightarrow T$ then plays the role of $\phi_{s}: Y_{s} \rightarrow X_{s}$, and the result is shown in this case.

On the other hand, if $P$ is non-trivial, we apply Case 1 to $\phi_{T}: Y_{T} \rightarrow T$ with $\Sigma$ replaced by $\psi_{T}^{-1}(\Sigma), \tau$ replaced by $\tau^{\prime}$, and $\sigma$ replaced by $\sigma_{1}^{\prime}$. Doing so we obtain a cover $\psi: X_{1} \rightarrow T$ that is étale away from $\phi_{T}^{-1}(\Sigma)$, linearly disjoint from $\phi_{T}: Y_{T} \rightarrow$ $T$, and such that the normalized pullback $\phi_{1}: Y_{1} \rightarrow X_{1}$ of $\phi_{T}$ via $\psi$ contains at least one point $\tau_{1}$ in $\psi^{-1}\left(\tau^{\prime}\right)$ that is unbranched in $\phi_{1}$. The cover $\phi_{1}: Y_{1} \rightarrow X_{1}$ is the normalized pullback of $\phi: Y \rightarrow X$ via $\psi_{1}=\psi_{T} \circ \psi$ because $\phi_{T}$ is the normalized pullback of $\phi$ via $\psi_{T}$ and $\phi_{1}$ is the normalized pullback of $\phi_{T}$ via $\psi$. Similarly, $X_{1}$ is linearly disjoint from $Y$ over $X$. Since $\psi_{1}\left(\tau_{1}\right)=\psi_{T} \circ \psi\left(\tau_{1}\right)=\psi_{T}\left(\tau^{\prime}\right)=\tau$, the cover $\psi_{1}$ has the asserted properties.

Case 3. The general case when $s=1$.

Applying Lemma 1 to the cover $\phi: Y \rightarrow X$ with $t=3$ and $\Delta=\{\tau\}$, there exists a cover $\pi: Z \rightarrow X$ of degree at least 3 that is linearly disjoint from $\phi: Y \rightarrow X$, is étale away from $\Delta$, and such that $\left|\pi^{-1}(\Delta)\right| \geq 3$. Let $Y_{Z}$ be the normalization of $Y \times_{X} Z$; this is irreducible because of linear disjointness. Hence the induced map $\phi_{Z}: Y_{Z} \rightarrow Z$ is $G$-Galois. Moreover, for any two points $\tau_{1}, \tau_{2} \in \pi^{-1}(\tau)$, the sets of inertia groups of $\phi_{Z}$ over $\tau_{1}$ and over $\tau_{2}$ are the same since $\pi$ is Galois. The result follows by applying Case 2 to the cover $Y_{Z} \rightarrow Z$, with $\Sigma$ replaced by $\pi^{-1}(\Sigma)$ and $\tau, \sigma_{1}$, and $\sigma_{2}$ replaced by any three points in $\pi^{-1}(\tau)$.

\section{Solving Embedding PROBlems With Restricted BRANCH LOCUS}

The goal of this section is to prove Theorem 5 which is a strengthening of the main theorem of [Ha99] (Theorem 5.4 of that manuscript) concerning embedding problems for branched covers over an algebraically closed field. That result gave a bound on the number of additional branch points that are needed in order to solve a given embedding problem. In the version we prove below, we also show that the positions of the additional branch points can be specified in advance (unlike Ha99, Theorem 5.4], which allowed only one of these additional points to be specified). 
In order to prove this strengthening, we will build upon the assertion of Theorem 5.4 of [Ha99, proceeding in two steps. First, we allow the additional branch points to move, obtaining a family of solutions to the given embedding problem each with the same number of additional branch points. Second, we show that the additional branch points can be specialized to the desired locations, while preserving the irreducibility and separability of the cover. The assertions needed to carry out these two steps appear in the next two propositions.

As before, $k$ is an algebraically closed field of characteristic $p>0$.

Proposition 3. Let $G$ be a finite group, let $X$ be a smooth connected projective $k$-curve, and let $f: Y \rightarrow X$ be a G-Galois cover with branch locus $B$. Write $B$ as a disjoint union $B_{0} \cup B_{1}$, where $B_{0}=\left\{\xi_{1}, \ldots, \xi_{r}\right\}$ is a set of $r>0$ distinct $k$-points.

(a) Then there is an integral affine $k$-variety $T$, a dominating morphism $\pi=$ $\left(\pi_{1}, \ldots, \pi_{r}\right): T \rightarrow X^{r}$, and a G-Galois cover $\tilde{f}: \tilde{Y} \rightarrow X \times T$, such that the fibre over each $\tau \in T$ is a $G$-Galois cover of $X$ with branch locus $B_{\tau} \cup B_{1}$, where $B_{\tau}:=\left\{\pi_{1}(\tau), \ldots, \pi_{r}(\tau)\right\}$ is disjoint from $B_{1}$, and such that for some $\tau_{0} \in \pi^{-1}\left(\xi_{1}, \ldots, \xi_{r}\right)$, the fibre over $\tau_{0}$ is isomorphic to the given $G$-Galois branched cover $Y \rightarrow X$.

(b) Moreover, if $H$ is a quotient of $G$ and $Y \rightarrow X$ dominates an $H$-Galois cover $g: Z \rightarrow X$ that is unramified over $B_{0}$, then the family $\tilde{f}: \tilde{Y} \rightarrow X \times T$ may be chosen to dominate the trivial family $\tilde{g}:=g \times$ id $: Z \times T \rightarrow X \times T$.

Proof. At each point $\xi_{i}$ of $X$, choose a uniformizing parameter $u_{i} \in \mathcal{O}_{X, \xi_{i}}$. Embed the tensor square of $\mathcal{O}_{X, \xi_{i}}$ over $k$ into the local ring of $X \times X$ at $\left(\xi_{i}, \xi_{i}\right)$. For short, we write $x_{i}, t_{i} \in \mathcal{O}_{X \times X,\left(\xi_{i}, \xi_{i}\right)}$ for the elements identified with $u_{i} \otimes 1,1 \otimes u_{i}$ respectively, and we also identify these elements with their images in the complete local ring $\hat{\mathcal{O}}_{X \times X,\left(\xi_{i}, \xi_{i}\right)}$. Consider the inclusion of complete local rings $\iota_{i}: \hat{\mathcal{O}}_{X, \xi_{i}} \hookrightarrow$ $\hat{\mathcal{O}}_{X \times X,\left(\xi_{i}, \xi_{i}\right)}$ given by $u_{i} \mapsto x_{i}-t_{i}$. With respect to $\iota_{i}$, for each point $\eta \in Y$ lying over $\xi_{i}$ we may form the tensor product $A_{\eta}:=\hat{\mathcal{O}}_{Y, \eta} \otimes_{\hat{\mathcal{O}}_{X, \xi_{i}}} \hat{\mathcal{O}}_{X \times X,\left(\xi_{i}, \xi_{i}\right)}$; here we regard $\hat{\mathcal{O}}_{Y, \eta}$ as an $\hat{\mathcal{O}}_{X, \xi_{i}}$-algebra via $f$. Then $A_{i}:=\prod_{f(\eta)=\xi_{i}} A_{\eta}$ is a $G$-Galois algebra over $\hat{\mathcal{O}}_{X \times X},\left(\xi_{i}, \xi_{i}\right)$ which is ramified precisely over the prime ideal $\left(x_{i}-t_{i}\right)$ of $\hat{\mathcal{O}}_{X \times X},\left(\xi_{i}, \xi_{i}\right)$. Moreover the fibre of its spectrum mod $t_{i}$ is isomorphic to the pullback of $Y \rightarrow X$ over $\hat{\mathcal{O}}_{X, \xi_{i}}$ as a $G$-Galois cover. (Here $A_{i}$ is the induced algebra $\operatorname{Ind}_{G_{\eta}}^{G} A_{\eta}$, where $G_{\eta}$ is the inertia group at $\eta$.)

Let $R^{*}=k\left[\left[t_{1}, \ldots, t_{r}\right]\right]$, which we identify with the complete local ring of $X^{r}$ at the point $\underline{\xi}:=\left(\xi_{1}, \ldots, \xi_{r}\right) \in X^{r}$ and whose fraction field will be denoted by $F^{*}$. For each $i$ write $R_{i}^{*}=\hat{\mathcal{O}}_{X \times X^{r},\left(\xi_{i}, \underline{\xi}\right)}$ and let $A_{i}^{*}=A_{i} \otimes_{\hat{\mathcal{O}}_{X \times X,\left(\xi_{i}, \xi_{i}\right)}} R_{i}^{*}$; here we regard $R_{i}^{*}$ as a module over $\hat{\mathcal{O}}_{X \times X,\left(\xi_{i}, \xi_{i}\right)}$ via the map $X \times X^{r} \rightarrow X \times X$ that projects $X^{r}$ onto its $i$-th factor. Since $r>0$, the curve $U=X-B_{0}$ is affine and may be written as $U=\operatorname{Spec} R_{U}$. Since the morphism $f$ is finite, it is affine, and hence $f^{-1}(U) \subset Y$ is affine. Let $A_{U}$ be its ring of functions, and let $A_{U}^{*}=A_{U} \otimes_{R_{U}} R_{U}^{*}$, where $R_{U}^{*}=R_{U}\left[\left[t_{1}, \ldots, t_{r}\right]\right]$. The $\bmod \left(t_{1}, \ldots, t_{r}\right)$-reductions of $A_{U}^{*}$ and $A_{i}^{*}$ can be identified with $A_{U}$ and $A_{i} / t_{i} A_{i}$, and so we obtain isomorphisms between the $G$-Galois algebras that these reductions induce over $\hat{\mathcal{K}}_{X, \xi_{i}}$, the fraction field of $\hat{\mathcal{O}}_{X, \xi_{i}}$. Writing $\hat{\mathcal{K}}_{X, \xi_{i}}^{*}=\hat{\mathcal{K}}_{X, \xi_{i}}\left[\left[t_{1}, \ldots, t_{r}\right]\right]$, we thus obtain an identification $A_{U}^{*} \otimes_{R_{U}^{*}} \hat{\mathcal{K}}_{X, \xi_{i}}^{*}=A_{i}^{*} \otimes_{R_{i}^{*}} \hat{\mathcal{K}}_{X, \xi_{i}}^{*}$ of $G$-Galois étale algebras for each $i$. 
We now apply the patching result Theorem 3.2.8 of Ha03b in the context of the category of $G$-Galois covers (see also General Principle 2.2.4 of that paper). As a consequence, we obtain a $G$-Galois branched cover $Y^{*} \rightarrow X^{*}:=X \times_{k} R^{*}$ whose restrictions to $\operatorname{Spec} R_{U}^{*}$ and to $\operatorname{Spec} R_{i}^{*}$ respectively agree with $\operatorname{Spec} A_{U}^{*}$ and Spec $A_{i}^{*}$, and whose closed fibre is isomorphic to $Y \rightarrow X$. The cover is branched at the points of $B_{1}^{*}:=B_{1} \times_{k} R^{*}$ and at $r$ distinct $R^{*}$-points $\xi_{1}^{*}, \ldots, \xi_{r}^{*}$. Here $\xi_{i}^{*}$ is the pullback to $X^{*}=X \times_{k} \mathcal{O}_{X^{r}, \xi}$ of the $X^{r}$-point $\xi_{i}^{\Delta}$ of $X \times X^{r}$, where $\xi_{i}^{\Delta}$ is the $X^{r}$-point at which the first coordinate (in $X$ ) is equal to the $i$ th entry of the second coordinate. More formally, if $\operatorname{pr}_{i}: X^{r} \rightarrow X$ is the $i$ th projection, then $\xi_{i}^{\Delta}$ is the inverse image of the diagonal $\Delta \subset X \times X$ under $\operatorname{id}_{X} \times \mathrm{pr}_{i}: X \times X^{r} \rightarrow X \times X$.

We claim that $Y^{*}$ is smooth over $R^{*}$. Namely, $Y^{*}$ is projective over $R^{*}$ since $X^{*}$ is. Hence if the singular locus of $Y^{*}$ is a (necessarily closed) non-empty subset, its image in $\operatorname{Spec}\left(R^{*}\right)$ contains the closed point. But then this point is in the image of the singular locus of $Y$ over $k$, contradicting the smoothness of $Y$ and proving the claim.

Note that in the situation of part (b), $Y^{*} \rightarrow X^{*}$ dominates the $H$-Galois cover $g^{*}=g \times \operatorname{id}_{\text {Spec } R^{*}}: Z^{*}:=Z \times_{k} R^{*} \rightarrow X^{*}$, since it does over $R_{U}^{*}, R_{i}^{*}$, and $\hat{\mathcal{K}}_{X, \xi_{i}}^{*}$, compatibly, and since the patching assertion of [Ha03b, Theorem 3.2.8] is an equivalence of categories.

Let $X^{\prime}=$ Spec $S$ be an affine open neighborhood of $\left\{\xi_{1}, \ldots, \xi_{r}\right\}$ in $X$. We may regard $S^{\otimes r}$ as a subring of the local ring of $X^{r}$ at $\underline{\xi}$ and hence as a subring of $R^{*}$. The $G$-Galois cover $Y^{*} \rightarrow X^{*}$ is defined by equations involving only finitely many elements of $R^{*}$. The $S^{\otimes r}$-subalgebra $\tilde{R} \subset R^{*}$ generated by these elements is of finite type, and the $G$-Galois cover $Y^{*} \rightarrow X^{*}$ is induced by the $G$-Galois cover $\tilde{Y} \rightarrow X \times T$, where $T=\operatorname{Spec}(\tilde{R})$. Here $\tilde{R}$ is a domain and $T$ is integral. The inclusion $S^{\otimes r} \hookrightarrow \tilde{R}$ corresponds to a morphism $T \rightarrow X^{\prime r}$; composing this with the embedding $X^{\prime r} \hookrightarrow X^{r}$ yields a morphism $\pi: T \rightarrow X^{r}$.

Possibly after enlarging the choice of $\tilde{R}$ by adjoining finitely many elements of $R^{*}$ (which are inverses of elements of $\tilde{R}$ ), we may assume that the above properties of $Y^{*} \rightarrow X^{*}$ descend to $\tilde{Y} \rightarrow X \times T$. Specifically, since $Y^{*}$ is smooth over $R^{*}$, we may choose $\tilde{Y}$ so as to be smooth over $T$. Similarly, we may choose $\tilde{Y} \rightarrow X \times T$ so that its fibre over $X \times\left\{\tau_{0}\right\}$ is isomorphic to $Y \rightarrow X$, where $\tau_{0} \in T$ is the image of the closed point of Spec $R^{*}$ (i.e. $\tau_{0}$ corresponds to the contraction of the maximal ideal of $R^{*}$ under the inclusion $\tilde{R} \hookrightarrow R^{*}$ ). Moreover we may choose $\tilde{Y} \rightarrow X \times T$ so that its branch locus consists of $\tilde{B}_{1}:=B_{1} \times T$ together with $r$ distinct $T$-points $\tilde{\xi}_{i}$, where $\tilde{\xi}_{i}: T \rightarrow X \times T$ is the pullback to $X \times T$, via $\pi: T \rightarrow X^{r}$, of the $X^{r}$-point $\xi_{i}^{\Delta}$ of $X \times X^{r}$ defined above. (Thus the image of $\tilde{\xi}_{i}$, which is a closed subset of $X \times T$ isomorphic to $T$, is a component of the branch locus of $\tilde{Y} \rightarrow X \times T$.) In addition we may assume that the loci $\tilde{\xi}_{i}$ are pairwise disjoint and are disjoint from $\tilde{B}_{1}$. Finally, in part (b), we may assume that $\tilde{Y} \rightarrow X \times T$ dominates the $H$-Galois cover $\tilde{g}=g \times \operatorname{id}_{T}: Z \times T \rightarrow X \times T$.

The morphism $\pi$ is dominating since $S^{\otimes r} \hookrightarrow \tilde{R}$ is an inclusion. Write $\pi_{i}=\operatorname{pr}_{i} \circ \pi$ : $T \rightarrow X$, so $\pi=\left(\pi_{1}, \ldots, \pi_{r}\right): T \rightarrow X^{r}$. Let $p: X \times X^{r} \rightarrow X$ denote projection onto the first factor $X$. Then $p \circ \xi_{i}^{\Delta}=\operatorname{pr}_{i}: X^{r} \rightarrow X$. Also $\xi_{i}^{\Delta} \circ \pi=\left(\operatorname{id}_{X} \times \pi\right) \circ \tilde{\xi}_{i}: T \rightarrow$ $X \times X^{r}$, since $\tilde{\xi}_{i}$ is the pullback of $\xi_{i}^{\Delta}$. Thus $\pi_{i}=\operatorname{pr}_{i} \circ \pi=p \circ\left(\operatorname{id}_{X} \times \pi\right) \circ \tilde{\xi}_{i}: T \rightarrow X$. Also, $\Delta \circ \operatorname{pr}_{i}=\left(\operatorname{id}_{X} \times \operatorname{pr}_{i}\right) \circ \xi_{i}^{\Delta}: X^{r} \rightarrow X \times X$, where $\Delta: X \rightarrow X \times X$ is the 
diagonal map. So $\tilde{\xi}_{i}=\left(\pi_{i}, \mathrm{id}_{T}\right)$, and hence the restriction of $\tilde{\xi}_{i}$ to the fiber over $\tau$ is $\left.\pi_{i}\right|_{\tau}: \tau \rightarrow X$ if we identify $X \times \tau$ with $X$.

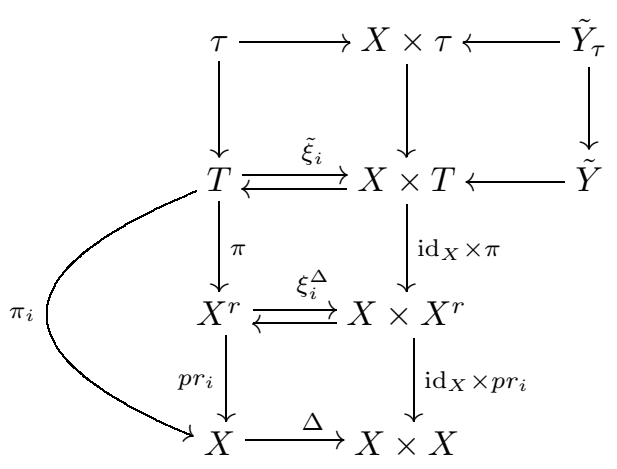

So for each $\tau \in T$, the corresponding fibre of $\tilde{Y} \rightarrow X \times T$ is branched at $B_{1}$ and at the $r$ points $\pi_{i}(\tau)$ of $X$ which are respectively the restrictions of the $T$-points $\tilde{\xi}_{i}$ of $X \times T$. Since $\tilde{\xi}_{i}$ pulls back to $\xi_{i}^{*}$ under Spec $R^{*} \rightarrow T$ and since $\xi_{i}^{*}$ restricts to $\xi_{i}$ on the closed fibre of $X^{*}=X \times_{k} R^{*}$, we have that $\pi_{i}\left(\tau_{0}\right)=\xi_{i}$. Finally, the fibres of $\tilde{Y} \rightarrow X \times T \rightarrow T$ are connected by Zariski's Connectedness Theorem (see [Ht, III, Exercise 11.4] or [EGA III.1, Théorème 4.3.1]), since the morphism is projective and the fibre over $\tau_{0}$ is the connected curve $Y$.

Proposition 4. In the situation of Proposition $3(\mathrm{~b})$, assume that $N:=\operatorname{ker}(G \rightarrow$ $H)$ has order prime to $p$. Let $B^{\prime}=\left\{\xi_{1}^{\prime}, \ldots, \xi_{r}^{\prime}\right\}$ be a set of $r$ distinct $k$-points of $X$ that is disjoint from $B_{1}$. Then there is a $G$-Galois cover $f^{\prime}: Y^{\prime} \rightarrow X$ that has branch locus $B^{\prime} \cup B_{1}$ and dominates $g: Z \rightarrow X$.

Proof. Let $\tilde{f}: \tilde{Y} \rightarrow X \times T$ be as in the conclusion of Proposition B(b). If $\underline{\xi}^{\prime}:=$ $\left(\xi_{1}^{\prime}, \ldots, \xi_{r}^{\prime}\right)$ is in the image of the dominating morphism $\pi: T \rightarrow X^{r}$, then the conclusion follows from the properties of $\tilde{f}$ and its fibres. More generally, we proceed as follows:

Since $T$ is an affine $k$-variety, we may regard $T$ as a closed subset of some $\mathbb{A}_{k}^{n}$. The graph $\Gamma \subset T \times X^{r} \subset \mathbb{A}_{k}^{n} \times X^{r}$ of $\pi$ is isomorphic to $T$ by the first projection map, so replacing $T$ by $\Gamma$ we may assume that $T \subset \mathbb{A}_{k}^{n} \times X^{r}$ and that $\pi$ is the second projection. Let $\bar{T}$ be the closure of $T$ in $\mathbb{P}_{k}^{n} \times X^{r}$ and let $\bar{\pi}: \bar{T} \rightarrow X^{r}$ be the second projection map. Since the projective morphism $\bar{\pi}$ extends $\pi: T \rightarrow X^{r}$, it is dominating and hence surjective. Let $\tau_{1} \in \bar{T}$ be a point that lies over $\underline{\xi}^{\prime} \in X^{r}$. Since $T$ is integral, there is an integral curve $\bar{C}_{0}$ in $\bar{T}$ that passes through $\tau_{0}$ and $\tau_{1}$. Let $C_{0}=\bar{C}_{0} \cap T$. Thus $\bar{C}_{0}$, being integral and closed in $\bar{T}$, is the closure of $C_{0}$ in $\bar{T}$.

The normalization of $X \times \bar{T}$ in the function field of $\tilde{Y}$ is a $G$-Galois branched cover $\tilde{Y}_{\bar{T}} \rightarrow Z \times \bar{T} \rightarrow X \times \bar{T}$ whose restriction to $X \times T$ is $\tilde{Y} \rightarrow Z \times T \rightarrow X \times T$. The pullback of this cover via $X \times \bar{C}_{0} \rightarrow X \times \bar{T}$ is a $G$-Galois branched cover $\tilde{Y}_{\bar{C}_{0}} \rightarrow Z \times \bar{C}_{0} \rightarrow X \times \bar{C}_{0}$ whose restriction to $X \times C_{0}$ is branched only over $B_{1} \times C_{0}$ and at the $C_{0}$-points $\xi_{i, C_{0}}:=\left(\pi_{i}, \mathrm{id}_{C_{0}}\right): C_{0} \rightarrow X \times C_{0}$ for $i=1, \ldots, r$ (where, as before, $\pi_{i}: T \rightarrow X$ is the composition of $\pi: T \rightarrow X^{r}$ with the $i$ th projection). Let $\bar{C}$ be the normalization of the curve $\bar{C}_{0}$, and let $\tilde{Y}_{\bar{C}} \rightarrow Z \times \bar{C} \rightarrow X \times \bar{C}$ be the normalized pullback of $\tilde{Y}_{\bar{C}_{0}} \rightarrow Z \times \bar{C}_{0} \rightarrow X \times \bar{C}_{0}$ via $X \times \bar{C} \rightarrow X \times \bar{C}_{0}$. So $\tilde{Y}_{\bar{C}} \rightarrow X \times \bar{C}$ is unramified away from $B_{1} \times \bar{C}$, the $\bar{C}$-points $\xi_{i, \bar{C}}:=\left(\bar{\pi}_{i}, \mathrm{id}_{\bar{C}}\right)$, and 
$X \times S$, where $S \subset \bar{C}$ is the finite set of points whose image in $\bar{C}_{0}$ does not lie in $C_{0}$. (Here $\bar{\pi}_{i}: \bar{C} \rightarrow X$ denotes the composition of the normalization map $\bar{C} \rightarrow \bar{C}_{0}$ with the map $\operatorname{pr}_{i} \circ \bar{\pi}: \bar{C}_{0} \rightarrow X$.) Hence the $N$-Galois cover $\tilde{Y}_{\bar{C}} \rightarrow Z \times \bar{C}$ is unramified away from $g^{-1}\left(B_{1}\right) \times \bar{C}$, the pullbacks $\left(g \times \operatorname{id}_{\bar{C}}\right)^{*}\left(\xi_{i, \bar{C}}\right)$ to $Z \times \bar{C}$ for $i=1, \ldots, r$, and $Z \times S$.

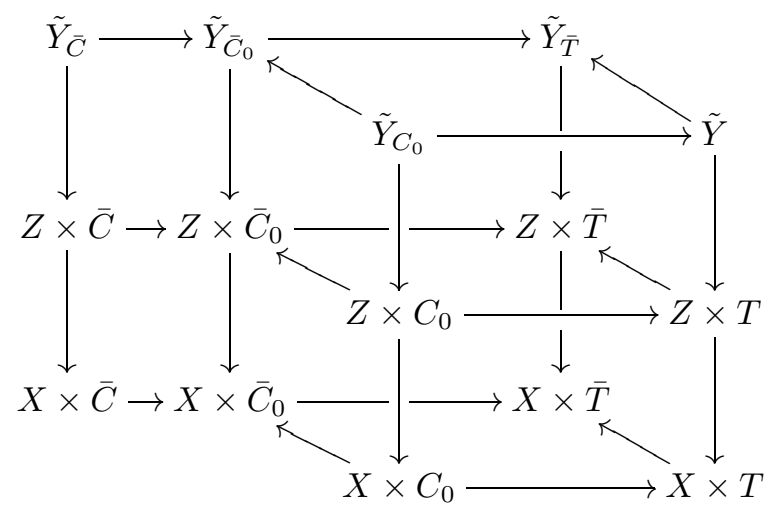

Let $m$ be the least common multiple of the ramification indices of $\tilde{Y}_{\bar{C}} \rightarrow Z \times \bar{C}$ over the generic points of $Z \times S$. Thus $m$ is prime to $p$, since the order of $N$ is prime to $p$. Choose a rational function $f$ on $\bar{C}$ that has a simple pole at each point of $S$, and possibly other poles. Adjoining an $m$-th root of $f$ and normalizing yields a cyclic branched cover $\bar{C}^{\prime} \rightarrow \bar{C}$ of degree $m$ that is totally ramified at the points of $S$ (and possibly elsewhere). Then by Abhyankar's Lemma, the normalized pullback $\tilde{Y}_{\bar{C}^{\prime}} \rightarrow Z \times \bar{C}^{\prime}$ of $\tilde{Y}_{\bar{C}} \rightarrow Z \times \bar{C}$ is unramified at the generic points of $Z \times S^{\prime}$, where $S^{\prime} \subset \bar{C}^{\prime}$ is the inverse image of $S \subset \bar{C}$. So by Purity of Branch Locus ( $\mathrm{Za}$, Proposition 2] or [Na, 41.1]), $\tilde{Y}_{\bar{C}^{\prime}} \rightarrow Z \times \bar{C}^{\prime}$ is branched only over $g^{-1}\left(B_{1}\right) \times \bar{C}^{\prime}$ and at the pullbacks $\left(g \times \operatorname{id}_{\bar{C}^{\prime}}\right)^{*}\left(\xi_{i, \bar{C}^{\prime}}\right)$ to $Z \times \bar{C}^{\prime}$ for $i=1, \ldots, r$, where $\xi_{i, \bar{C}^{\prime}}$ is the pullback of $\xi_{i, \bar{C}}$ under $\bar{C}^{\prime} \rightarrow \bar{C}$.

Let $\tau^{\prime} \in \bar{C}^{\prime}$ be a point mapping to $\tau_{1} \in \bar{C}_{0}$ via $\bar{C}^{\prime} \rightarrow \bar{C} \rightarrow \bar{C}_{0}$. The components of $B_{1} \times \bar{C}^{\prime}$ and the $\bar{C}^{\prime}$-points $\xi_{i, \bar{C}^{\prime}}$ are each smooth over $\bar{C}^{\prime}$, as are their inverse images under $Z \times \bar{C}^{\prime} \rightarrow X \times \bar{C}^{\prime}$. In the fibre of $X \times \bar{C}^{\prime}$ over $\tau^{\prime}$, these loci specialize to distinct points of $X$ (viz. to the points of $B_{1}$ and $B^{\prime}$ respectively); hence their inverse images in $Z \times \bar{C}^{\prime}$ are also disjoint over $\tau^{\prime}$. So the union of these inverse images is smooth over $\tau^{\prime}$, and there is an affine neighborhood $C^{\prime} \subset \bar{C}^{\prime}$ of $\tau^{\prime}$ such that the (tame) branch locus of the restriction $\tilde{Y}_{C^{\prime}} \rightarrow Z \times C^{\prime}$ of $\tilde{Y}_{\bar{C}^{\prime}} \rightarrow Z \times \bar{C}^{\prime}$ is smooth over $\bar{C}^{\prime}$. By Abhyankar's Lemma (in the form [GM, Theorem 2.3.2] or SGA1, Proposition 5.5]), the cover $\tilde{Y}_{C^{\prime}} \rightarrow Z \times C^{\prime}$ is a Kummer cover étale locally, with smooth branch locus, hence is smooth over $C^{\prime}$. So the fibre over $\tau^{\prime}$ is smooth over $k$.

The intersection of $C^{\prime}$ with the inverse image of $C_{0}$ under $\bar{C}^{\prime} \rightarrow \bar{C}_{0}$ is a dense open subset of $C^{\prime}$. For each point $\tau$ in this dense open subset, the fibre of the projective morphism $\tilde{Y}_{C^{\prime}} \rightarrow C^{\prime}$ over $\tau$ is connected by the conclusion of Proposition 3. By Zariski's Connectedness Theorem [Ht, III, Exercise 11.4], it follows that each fibre of $\tilde{Y}_{C^{\prime}} \rightarrow C^{\prime}$ is connected, in particular the fibre over $\tau^{\prime}$. So the fibre of the $G$ Galois cover $\tilde{Y}_{C^{\prime}} \rightarrow X \times C^{\prime}$ over the point $\tau^{\prime} \in C^{\prime}$ is a $G$-Galois cover of $X$ with the desired properties. 
We recall the definition of relative rank of a subgroup of a finite group (see Section 2 of [Ha99]). Let $H$ be any finite group and let $E$ be a subgroup of $H$. A subset $S \subset E$ will be called a relative generating set for $E$ in $H$ if for every subset $T \subset H$ such that $E \cup T$ generates $H$, the subset $S \cup T$ also generates $H$. We define the relative rank of $E$ in $H$ to be the smallest non-negative integer $s:=\operatorname{rk}_{H}(E)$ such that there is a relative generating set for $E$ in $H$ consisting of $s$ elements. Thus every generating set for $E$ is a relative generating set, and so $0 \leq \operatorname{rk}_{H}(E) \leq \operatorname{rk}(E)$. Also, $\operatorname{rk}_{H}(E)=\operatorname{rk}(E)$ if $E$ is trivial or $E=H$, while $\operatorname{rk}_{H}(E)=0$ if and only if $E$ is contained in the Frattini subgroup $\Phi(H)$ of $H$ [Ha99, p. 122]. Also recall that $p(G)$ denotes the subgroup generated by the $p$-subgroups of a group $G$.

Theorem 5. Let $N$ be a normal subgroup of a finite group $G$, and let $H=G / N$. Let $\bar{N}=N / p(N)$ and $\bar{G}=G / p(N)$, and let $r=\operatorname{rk}_{\bar{G}}(\bar{N})$. Let $V \rightarrow U$ be an $H$-Galois étale cover of affine $k$-curves and let $\xi_{1}, \ldots, \xi_{r} \in U$ be distinct points. Then there is a G-Galois cover $W \rightarrow U$ branched only at $\xi_{1}, \ldots, \xi_{r} \in U$ such that $W / N \rightarrow U$ is isomorphic to $V \rightarrow U$ as an $H$-Galois cover.

Proof. We may identify $\bar{G} / \bar{N}$ with $H$. Let $X$ be the smooth completion of $U$, i.e., the unique smooth proper $k$-curve that contains $U$ as an affine dense open subset. Let $Z$ be the normalization of $X$ in the function field of $V$. Thus the inclusion $U \hookrightarrow X$ lifts to an inclusion $V \hookrightarrow Z$. Also let $B_{1}=X-U$, let $B_{0}=\left\{\xi_{1}, \ldots, \xi_{r}\right\}$, and let $B=B_{0} \cup B_{1}$. We claim that there is a $\bar{G}$-Galois cover $\bar{Y} \rightarrow X$ that dominates $Z \rightarrow X$ and is branched only at $B$ or, equivalently, a $\bar{G}$-Galois cover $\bar{W} \rightarrow U$ that dominates $V \rightarrow U$ and is branched only at $B_{0}$.

First suppose $r=0$. For some subgroup $\bar{G}_{0} \subset \bar{G}$, the cover $H$-Galois cover $V \rightarrow U$ is dominated by a $\bar{G}_{0}$-Galois étale cover $\bar{W} \rightarrow U$, because the fundamental group of the affine $k$-curve $U$ has cohomological dimension 1 [Se90, Proposition 1] and hence is projective (by [Se73, I, §3.4, Prop. 16 and §5.9, Prop. 45]). Thus $\bar{G} / \bar{N}=H=\bar{G}_{0} /\left(\bar{G}_{0} \cap \bar{N}\right)=\bar{G}_{0} \bar{N} / \bar{N}$, and so $\bar{G}$ is generated by $\bar{G}_{0}$ and $\bar{N}$. But $\bar{N}$ is contained in the Frattini subgroup of $\bar{G}$ because $r=0$. Hence $\bar{G}_{0}=\bar{G}$, and $\bar{W} \rightarrow U$ is as desired. (Cf. also [FJ, Proposition 22.5.9(a)].)

On the other hand, if $r \geq 1$, then by [Ha99, Theorem 5.4] there is a smooth connected $\bar{G}$-Galois cover $\bar{W}_{0} \rightarrow U$ branched at a set $B^{\prime}$ of $r$ points such that $\bar{W}_{0} / \bar{N} \rightarrow U$ is isomorphic to $V \rightarrow U$ as an $H$-Galois cover. Let $\bar{Y}_{0}$ be the normalization of $X$ in $\bar{W}_{0}$. So $Z=\bar{Y}_{0} / \bar{N}, Z \rightarrow X$ is unramified outside of $B_{1}:=X-U$, and $\bar{Y}_{0} \rightarrow X$ is unramified outside of $B_{1} \cup B^{\prime}$. Since $\bar{N}$ has order prime to $p$, Proposition 4 above asserts that there is a smooth connected $\bar{G}$-Galois branched cover $\bar{Y} \rightarrow X$ that is branched only at $B$ and that dominates $Z \rightarrow X$. This completes the proof of the claim.

Since $p(N)$ is a quasi-p-group and $B$ is non-empty, Theorem 4.4 of $[\mathrm{Ha} 03 \mathrm{a}$ asserts that the $\bar{G}$-Galois cover $\bar{Y} \rightarrow X$ is dominated by some smooth connected $G$-Galois branched cover $Y \rightarrow X$ that is also branched only over $B$. Since $\bar{Y} \rightarrow X$ dominates the $H$-Galois cover $Z \rightarrow X$, so does $Y \rightarrow X$, and hence the restriction $W \rightarrow U$ of $Y \rightarrow X$ over $U$ has the desired properties.

\section{MAIN THEOREM}

Using the results of the previous sections, we prove our main theorem that the fundamental group of any affine curve in characteristic $p>0$ is almost $\omega$-free. To do so we combine the "newly unbranched" points in the covers obtained in Section 2 with the main result of Section 3 to solve the induced embedding problem. 
Theorem 6. Let $k$ be an algebraically closed field of characteristic p, let $X$ be a smooth connected projective curve, let $\Sigma \subset X$ be a non-empty set of closed points in $X$, and let $C=X-\Sigma$. Then $\pi_{1}(C)$ is almost $\omega$-free.

Proof. Explicitly, we wish to show the following: Let $\mathcal{E}=\left(\alpha: \pi_{1}(C) \rightarrow G, f\right.$ : $\Gamma \rightarrow G)$ be an embedding problem for $\pi_{1}(C)$. Then there exists an open subgroup $H \subset \pi_{1}(C)$ such that $\alpha_{H}:=\left.\alpha\right|_{H}$ is surjective and such that the induced embedding problem $\mathcal{E}_{H}=\left(\alpha_{H}, f\right)$ has a proper solution.

Let $N=\operatorname{ker}(f)$, and write $\bar{N}=N / p(N)$ and $\bar{\Gamma}=\Gamma / p(N)$. Let $\tau \in \Sigma$ and let $s=\operatorname{rk}_{\bar{\Gamma}}(\bar{N})$ (see the definition of relative rank just before Theorem 5). Let $\phi: Y \rightarrow X$ be the pointed $G$-Galois cover corresponding to $\alpha$. By Proposition 2 there exists a cover $\psi_{s}: X_{s} \rightarrow X$ that is étale away from $\Sigma$, contains at least $s$ points $\left\{\tau_{1}, \tau_{2}, \ldots, \tau_{s}\right\} \subset X_{s}$ over $\tau \in X$, and whose normalized pullback $\phi_{s}: Y_{s} \rightarrow X_{s}$ of $\phi$ over $\psi_{s}$ is a $G$-Galois cover étale away from $\Delta:=\psi_{s}^{-1}(\Sigma)-\left\{\tau_{1}, \tau_{2}, \ldots, \tau_{s}\right\}$. Let $\Sigma_{s}=\psi_{s}^{-1}(\Sigma)$. Applying Theorem 5 to the étale loci of $\phi_{s}: Y_{s} \rightarrow X_{s}$, there exists a smooth connected $\Gamma$-Galois cover $Z_{s} \rightarrow X_{s}$ that is étale away from $\Delta \cup\left\{\tau_{1}, \ldots, \tau_{s}\right\}=$ $\Sigma_{s}$, such that $Z_{s} / N$ is isomorphic to $Y_{s}$ as $G$-Galois covers of $X_{s}$. Now, $\psi_{s}: X_{s} \rightarrow X$ is ramified at most over $\Sigma$, and the pullback $\phi_{s}: Y_{s} \rightarrow X_{s}$ of $\phi$ via $\psi_{s}$ is a $G$-Galois cover. Moreover, the $\Gamma$-Galois cover $Z_{s} \rightarrow X_{s}$ dominates $Y_{s} \rightarrow X_{s}$. After some choice of base point, $\psi_{s}: X_{s} \rightarrow X$ corresponds to a subgroup $H \subset \pi_{1}(X-\Sigma)$ and a surjection $\alpha_{H}: H \rightarrow G$. We then find that $\left.\alpha\right|_{H}=\alpha_{H}$ and that the induced embedding problem $\mathcal{E}_{H}=\left(\alpha_{H}, f\right)$ has a proper solution.

The situation in characteristic zero is somewhat different, because fundamental groups of curves in characteristic zero are finitely generated. Specifically, there is the following result:

Proposition 7. Let $X$ be a smooth connected projective curve of genus $g$ defined over an algebraically closed field $k$ of characteristic 0 and let $\Sigma$ be a non-empty finite collection of points on $X$. The group $\pi_{1}(X-\Sigma)$ is almost $\omega$-free if and only if $X-\Sigma$ is not isomorphic to the once or twice punctured projective line.

Proof. Suppose first that $X-\Sigma$ is not isomorphic to the once or twice punctured projective line. Thus $g \geq 0, r:=|\Sigma| \geq 1$, and either $g \geq 1$ or $r \geq 3$; hence $2 g+r \geq 3$. Let $\mathcal{E}=(\alpha: \Pi \rightarrow G, f: \Gamma \rightarrow G)$ be a finite embedding problem for $\pi_{1}(X-\Sigma)$. Choose a prime number $d$ that is greater than the order of $\Gamma$. The surjection $\alpha: \pi_{1}(X-\Sigma) \rightarrow G$ determines a $G$-Galois cover $Y \rightarrow X$ that is étale away from $\Sigma$. Pick $\tau \in \Sigma$ and set $\Sigma^{\prime}=\Sigma-\{\tau\}$. Thus $X-\Sigma^{\prime}$ is isomorphic either to the projective line with at least two points deleted or else to a dense open subset of a curve of genus at least one. Hence $\pi_{1}\left(X-\Sigma^{\prime}\right)$ has a quotient that is cyclic of order $d$, corresponding to a $d$-cyclic cover $\phi_{d}: Z_{d} \rightarrow X$ that is étale away from $\Sigma^{\prime}$. The unramified fibre $\phi_{d}^{-1}(\tau)$ contains at least $d$ points, and $\Sigma_{d}:=\phi_{d}^{-1}(\Sigma)$ contains at least $r-1+d>0$ points. Since $Z_{d}$ has genus at least $g$, the fundamental group $\Pi_{0}=\pi_{1}\left(Z_{d}-\Sigma_{d}\right) \subset \pi_{1}(X-\Sigma)$ is free of rank at least $2 g+(r-1+d)-1>d$. Also, $Z_{d} \rightarrow X$ is linearly disjoint from $Y \rightarrow X$ because they have no common subcovers, the degree of $Z_{d} \rightarrow X$ being a prime number greater than the degree of $Y \rightarrow X$. Thus $\left.\alpha\right|_{\Pi_{0}}: \Pi_{0} \rightarrow G$ is surjective. Since the rank of $\Pi_{0}$ is larger than the minimal number of generators of $\Gamma$, by [FJ, Proposition 17.7.3] there is a proper solution to the induced embedding problem $\mathcal{E}_{0}=\left(\left.\alpha\right|_{\Pi_{0}}: \Pi_{0} \rightarrow G, f: \Gamma \rightarrow G\right)$. Thus $\pi_{1}(X-\Sigma)$ is almost $\omega$-free. 
Conversely, suppose that $X-\Sigma$ is the once or twice punctured projective line over $k$. In the former case $X-\Sigma$ has no non-trivial étale covers, while in the latter case every finite étale cover is itself isomorphic to the twice punctured projective line. Thus any open subgroup $\Pi_{0}$ of $\Pi=\pi_{1}(X-\Sigma)$ is either trivial or has rank one. So given a finite embedding problem $\mathcal{E}=(\alpha: \Pi \rightarrow G, f: \Gamma \rightarrow G)$ for $\Pi$ such that $\Gamma$ is not cyclic, there is no proper solution to the induced embedding problem $\mathcal{E}_{0}$ for any open subset $\Pi_{0}$. Thus $\pi_{1}(X-\Sigma)$ is not almost $\omega$-free.

This result helps clarify the relationships among the conditions on a profinite group being free, $\omega$-free, and almost $\omega$-free. For infinitely generated profinite groups, every free group is $\omega$-free (i.e. every finite embedding problem has a proper solution) but not conversely, as noted in the introduction. Also, every infinitely generated $\omega$-free profinite group is (trivially) almost $\omega$-free, and again the converse fails, since the fundamental group of an affine curve in characteristic $p$ is almost $\omega$-free by Theorem [ 6 but is not $\omega$-free, as noted in the introduction. In contrast, finitely generated free profinite groups $\Pi$ are not $\omega$-free, since an embedding problem $\mathcal{E}=(\Pi \rightarrow G, \Gamma \rightarrow G)$ cannot have a proper solution if the rank of $\Gamma$ is greater than that of $\Pi$. Concerning the almost $\omega$-free condition, the above proposition has the following consequence:

Corollary 8. A free profinite group of finite rank $m$ is almost $\omega$-free if and only if $m>1$.

Proof. A free profinite group of finite rank $m \geq 0$ is isomorphic to the algebraic fundamental group of the complex projective line minus $m+1$ points. Thus the result follows from the proposition above.

Note that this corollary could alternatively be proved using the fact that an open subgroup of index $i$ in a free group of finite rank $m$ is itself free of finite rank $1+i(m-1)$ [RiZa, Theorem 3.6.2(b)]. Proposition 7 could then be deduced using the fact that the fundamental group of an $r$-punctured curve of genus $g$ (for $r>0)$ is free of rank $2 g+r-1$. On the other hand, while the proof given in RiZa, Theorem 3.6.2(b)] is purely group theoretic, that result can alternatively be deduced easily from the Riemann-Hurwitz formula using fundamental groups of affine curves in characteristic zero, just as Corollary 8 was deduced above from Proposition 7

\section{ACKNOWLEDGMENTS}

We thank Florian Pop for suggestions involving Section 3 of this paper and Moshe Jarden for suggested improvements to the exposition.

\section{REFERENCES}

[Bo] I. Bouw. Construction of covers in positive characteristic via degeneration. Proc. Amer. Math. Soc. 137 (2009), no. 10, 3169-3176. MR.2515387(2010g:14036)

[Do] A. Douady. Détermination d'un groupe de Galois. C. R. Acad. Sci. Paris 258 (1964), 5305-5308. MR0162796 (29:100)

[FJ] M. Fried, M. Jarden. "Field Arithmetic", second edition. Ergebnisse Math. series, 11, Springer-Verlag, 2000. MR2102046 (2005k:12003)

[EGA III.1] A. Grothendieck. "Éléments de géométrie algébrique" (EGA), Volume III, Part 1. Publ. Math. IHES, 11, 1961. MR0217085 (36:177c) 
[GM] A. Grothendieck, J.P. Murre. "The tame fundamental group of a formal neighborhood of a divisor with normal crossings on a scheme". Lecture Notes in Mathematics, 208, Springer-Verlag, 1971. MR0316453 (47:5000)

[SGA1] A. Grothendieck. "Revêtements étales et groupe fondamental" (SGA 1). Lecture Notes in Mathematics, 224, Springer-Verlag, Berlin-Heidelberg-New York, 1971. MR 0354651 (50:7129)

[GS] R. Guralnick, K. F. Stevenson. Prescribing ramification. Arithmetic fundamental groups and noncommutative algebra, Proc. Sympos. Pure Math., 70, Amer. Math. Soc., Providence, RI, 2002, 387-406. MR.1935415 (2003i:14031)

[Ha80] D. Harbater. Moduli of p-covers of curves, Communications in Algebra 8 (1980), 1095-1122. MR.579791 (82f:14010)

[Ha94] D. Harbater. Abhyankar's conjecture on Galois groups over curves. Inventiones Math. 117 (1994), 1-25. MR1269423 (95i:14029)

[Ha95] D. Harbater. Fundamental groups and embedding problems in characteristic $p$. In Recent Developments in the Inverse Galois Problem (M. Fried et al., eds.), AMS Contemporary Mathematics Series, 186, 1995, 353-369. MR1352282 (97b:14035)

[Ha99] D. Harbater. Embedding problems and adding branch points, in "Aspects of Galois Theory", London Mathematical Society Lecture Note series, 256, Cambridge University Press, 1999, 119-143. MR.1708604 (2000f:14042)

[Ha03a] D. Harbater. Abhyankar's conjecture and embedding problems, Crelle's Journal, 559 (2003), 1-24. MR1989642 (2004g:14031)

[Ha03b] D. Harbater. Patching and Galois theory. In Galois Groups and Fundamental Groups (L. Schneps, ed.), MSRI Publications series, 41, 2003, Cambridge University Press, 313-424. MR2012220 (2004j:14030)

[Ht] R. Hartshorne. "Algebraic geometry". Graduate Texts in Mathematics, 52, SpringerVerlag, 1977. MR0463157 (57:3116)

[Iw] K. Iwasawa. On solvable exensions of algebraic number fields. Annals of Math. (2) 58 (1953), 548-572. MR0059314 (15:509d)

[Ja] M. Jarden. On free profinite groups of uncountable rank. In Recent Developments in the Inverse Galois Problem (M. Fried et al., eds.), AMS Contemporary Mathematics Series, 186, 1995, 371-383. MR1352283 (96g:12003)

[Ku08] M. Kumar, Fundamental groups in positive characteristic, J. Algebra 319 (2008), no. 12, 5178-5207. MR2423823 (2009k:14043)

[Ku09] M. Kumar, The fundamental group of affine curves in positive characteristic, 2009 manuscript, available at arXiv:0903.4472.

[Na] M. Nagata. "Local rings". Interscience, 1962. MR0155856 (27:5790)

[Po] F. Pop. Étale Galois covers of affine smooth curves. The geometric case of a conjecture of Shafarevich. On Abhyankar's conjecture. Inventiones Math. 120 (1995), 555-578. MR1334484 (96k:14011)

[Os] B. Osserman. Linear series and the existence of branched covers. Compos. Math. 144 (2008), no. 1, 89-106. MR2388557 (2009b:14056)

[Pr] R. Pries. Wildly ramified covers with large genus. J. Number Theory 119 (2006), no. 2, 194-209. MR2250044 (2007c:14023)

[Ra] M. Raynaud. Revêtements de la droite affine en caractéristique $p>0$ et conjecture d'Abhyankar. Inventiones Math. 116 (1994), 425-462. MR1253200 (94m:14034)

[RiZa] L. Ribes, P. Zalesskii, Profinite groups, Springer-Verlag, 2000. MR.1775104 (2001k:20060)

[Se73] J.-P. Serre. "Cohomologie Galoisienne", 4th ed., Lecture Notes in Math., 5, Springer, 1973. MR0404227 (53:8030)

[Se90] J.-P. Serre. Construction de revêtements étales de la droite affine en caractéristique p. C. R. Acad. Sci. Paris 311 (1990), 341-346. MR1071640(92b:14008)

[Ta] A. Tamagawa. Finiteness of isomorphism classes of curves in positive characteristic with prescribed fundamental groups. J. Algebraic Geom. 13 (2004), no. 4, 675-724. MR.2073193 (2005c:14032)

[Za] O. Zariski. On the purity of the branch locus of algebraic functions. Proc. Nat. Acad. Sci. U.S.A. 44 (1958), 791-796. MR0095846 (20:2344) 
Department of Mathematics, University of Pennsylvania, Philadelphia, Pennsylvania 19104-6395

E-mail address: harbater@math.upenn.edu

Department of Mathematics, California State University, Northridge, California 91330

E-mail address: katherine.stevenson@csun.edu 